\title{
ArDM, a 1t liquid argon detector for dark matter searches
}

\author{
C. Amsler* \\ Physik-Institut der Universität Zürich \\ E-mail: claude.amsler@cern.ch
}

\begin{abstract}
Weak Interacting Massive Particles (WIMPs) are prime candidates for the dark matter in the universe. The ArDM experiment consists of a 1t liquid argon detector to search for WIMPs interacting elastically with argon nuclei. Both scintillation light and ionization charge from nuclear recoils are measured. The $128 \mathrm{~nm}$ light is reflected and shifted into the visible range by wavelength shifting foils and detected by $8 "$ photomultipliers immersed in the liquid. The charge will be detected above the liquid surface by a double stage large electron multiplier. The detector is being developed at CERN on the surface before being moved to an underground site. ArDM was filled for the first time in spring 2009 and operated for more than one month. Preliminary results lead to a light collection efficiency of about 0.3 photoelectrons / kev for nuclear recoils, which shows that our goal of reaching a detection threshold of $30 \mathrm{keV}$ can be achieved,
\end{abstract}

The 2009 Europhysics Conference on High Energy Physics,

July 16 - 222009

Krakow, Poland

\footnotetext{
* Speaker.

† On behalf the ArDM collaboration: Andrzej Soltan Institute (Warsaw), CIEMAT (Madrid), ETH-Zürich, Universities of Granada, Sheffield and Zürich.
} 
The current upper limit for the elastic cross section between weak interacting massive particles (WIMPs) and nucleons is around $4 \times 10^{-8} \mathrm{pb}$ [1]. The goal of ArDM is to improve on the sensitivity to WIMPs by more than one order of magnitude. We are constructing at CERN a $1 \mathrm{t}$ liquid argon detector using the two-phase technique to detect both the charge and the luminescence produced by the recoil nuclei following a WIMP interaction. We have studied ways to efficiently collect and detect the VUV light to reach a detection threshold of $30 \mathrm{keV}$ in argon and to suppress background from neutrons and electrons, in particular from the $\beta$-emitter ${ }^{39} \mathrm{Ar}$ isotope.

Charged particles lead to ionization and excitation of argon atoms forming excimers $\left(\mathrm{Ar}_{2}^{+}\right.$ and $\mathrm{Ar}_{2}^{*}$ ), with the lowest singlet and the triplet excited states decaying into the ground state (two independent atoms) by VUV photon emission in a narrow band around $128 \mathrm{~nm}$. Reabsorption by argon atoms is energetically suppressed. The singlet and the triplet states have different decay times, respectively $\tau_{1} \simeq 5$ ns and $\tau_{2} \simeq 1.6 \mu$ s in liquid [2]. However, impurities such as water, air and $\mathrm{CO}_{2}$ can absorb the VUV light and reduce $\tau_{2}$ [3]. The population ratio singlet/triplet depends on ionization density. For minimum ionizing projectiles, such as electrons and $\gamma$ 's, the ratio is $\approx 0.3$, while for $\alpha$ 's and nuclear recoils one finds a ratio of $\approx 4$ [2]. Hence nuclear recoils from WIMPs populate mostly the fast decaying singlet state. In addition, the ratio of scintillation to ionization yield is much higher for nuclear than for minimum ionizing particles. This is due to quick recombination which decreases the charge and enhances the luminescence. The higher ratio of light to charge production for nuclear recoils and the higher population of the fast decaying state can both be used to reduce background in WIMP searches.
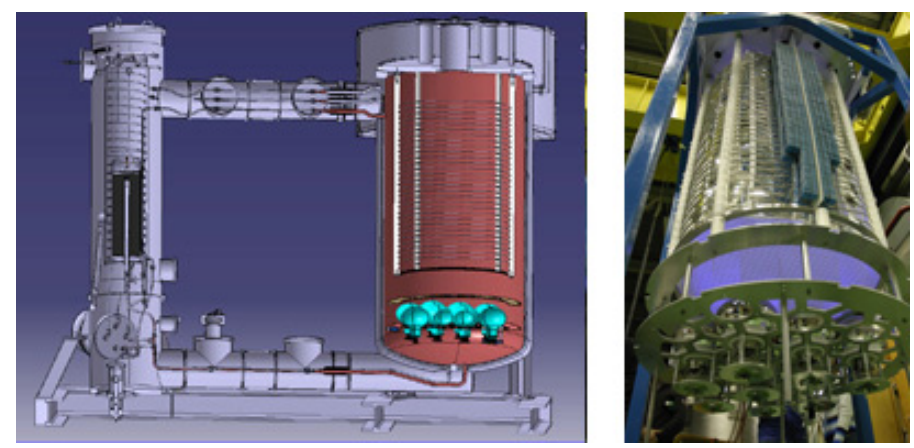

Figure 1: Left: sketch of the ArDM detector and its purification system on the left. Right: photograph of the detector showing the Greinacher HV divider, the WLS foils and the photomultiplier support mechanics.

A sketch of the ArDM detector, as it is now installed in building 182 at CERN (RE18experiment), is shown in fig. 1 (left). The working principle is as follows: in liquid argon a WIMP collision leading to $30 \mathrm{keV}$ nuclear recoils produces about $400 \mathrm{VUV}$ (128 $\mathrm{nm}$ ) photons, together with a few free electrons. The latter drift in a strong vertical electric field and are detected in the gas phase by a large electron multiplier (LEM) above the surface of the liquid, while the VUV scintillation light from argon is shifted into blue light by tetraphenylbutadiene (TPB) evaporated on Tetratex foils and detected by cryogenic photomultipliers (PMT) at the bottom of the vessel. Fifteen Tetratex sheets $\left(120 \times 25 \mathrm{~cm}^{2}\right)$ are coated to cover the cylindrical walls inside the electric field shaping rings. The light detection system consists of 14 photomultipliers ( 8 " hemispherical Hamamatsu R5912-MOD manufactured with Pt-underlays) at the bottom of the vessel. 
The PMT glass is coated with a thin layer of a transparent TPB-paraloid compound to increase the light collection efficiency. Figure 1 (right) shows the detector inside the vessel.

In parallel to the construction of ArDM we optimized the light output and collection efficiency for charged particles and neutrons. We used a small cylindrical cell containing liquid argon (78 $\mathrm{mm}$ high, $74 \mathrm{~mm}$ in diameter) viewed by two PMTs (Hamamatsu low temperature photomultipliers R6091-02MOD). A wavelength shifter ( $1 \mathrm{mg} / \mathrm{cm}^{2}$ of TPB) was evaporated on reflective foils covering the internal wall. ${ }^{210} \mathrm{~Pb}$ radioactive source producing $5.3 \mathrm{MeV} \alpha$ 's and up to $1.2 \mathrm{MeV}$ electrons was held on a stick in the middle of the active volume. Measurements with neutrons were obtained by placing a $370 \mathrm{MBq}$ Am-Be-source next to the experimental setup. The $5.4 \mathrm{MeV} \alpha$ 's from americium are absorbed by beryllium, producing $2-12 \mathrm{MeV}$ neutrons. The source produced about $2 \times 10^{4} \mathrm{n} / \mathrm{s}$. It was contained in a high density polyethylene shielding block with two apertures, one for the neutrons, the second for the $\gamma$ 's detected in coincidence in a $\mathrm{NaI}(\mathrm{Tl})$ detector. The neutron flux at the target position was around $30 \mathrm{~s}^{-1}$.
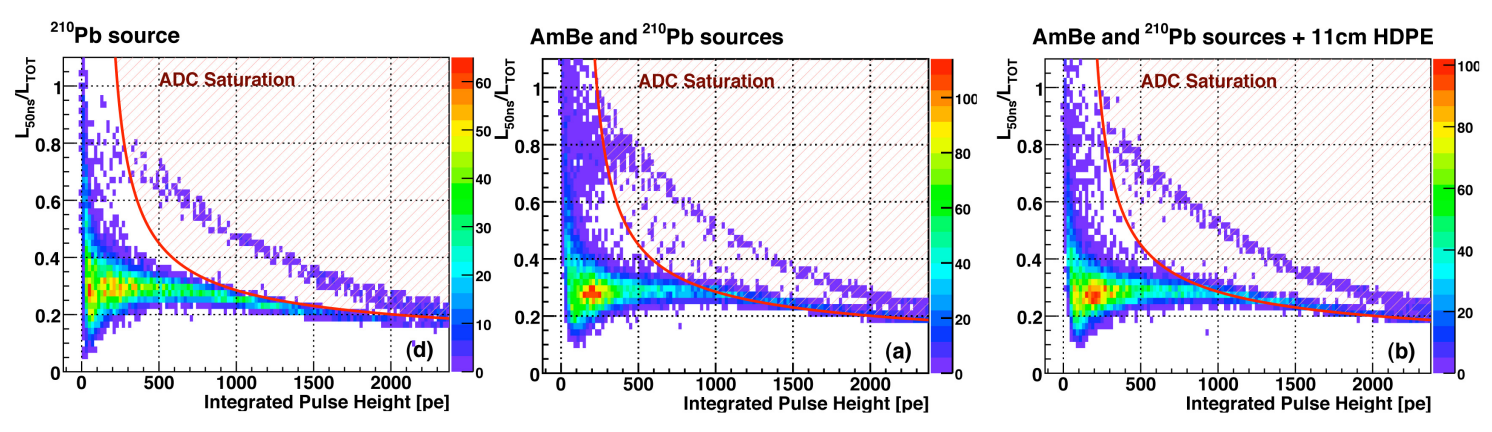

Figure 2: Ratio $r=L_{50 n s} / L_{T O T}$ of fast to total amplitude for the Pb-sources (left), with the additional AmBe-source (center), and with $11 \mathrm{~cm}$ of polyethylene absorber (right). Data points above the red line are affected by ADC saturation from $\alpha$ 's.

As pointed out before, heavily ionizing particles populate mostly the fast decaying singlet states. We therefore define the fraction $r$ of fast $(<50 \mathrm{~ns})$ component to enhance nuclear recoils. Figure 2 shows the measured ratio $r$. The accumulation of events around $r=0.3$ is due to electrons. The signal from recoil nuclei associated with neutrons is shown in fig. 2 around $r=0.8$. This can be proven by inserting a $11 \mathrm{~cm}$ thick polyethylene absorber, while a $4 \mathrm{~cm}$ lead sheet has no effect (not shown).

Even small neutron fluxes around $1 \mathrm{MeV}$ are potentially dangerous, since the neutron-argon cross section is some 18 orders of magnitude larger than for WIMPs. It is therefore essential to investigate the response of the dark matter detector to neutrons as a function of recoil energy. A decisive advantage of monoenergetic neutrons over radioactive sources (such as Am-Be) is the known incident energy from which the energy transferred to the nucleus can be calculated by measuring the neutron elastic scattering angle. We have therefore ordered a neutron source from NSD-Fusion GmbH. The source will deliver monoenergetic $2.45 \mathrm{MeV}$ neutrons $\left(10^{7} \mathrm{~s}^{-1}\right)$ from the reaction $\mathrm{dd} \rightarrow \mathrm{He}^{3} \mathrm{n}$. In the $1 \mathrm{t}$ detector the probability for two or more neutron interactions is large enough to be measured precisely. This fraction can be measured with the dd-source and used to reduce the neutron background during WIMP searches, since WIMP interactions do not lead to 
multiple scattering events.

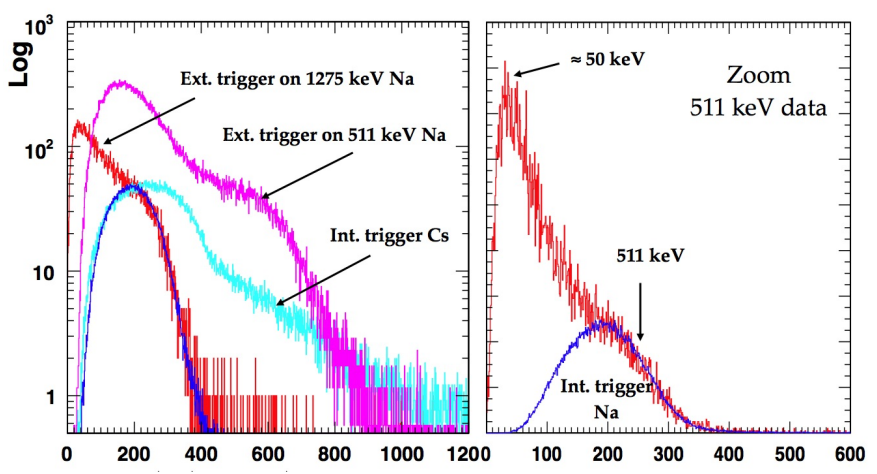

Figure 3: Preliminary $\gamma$-spectrum from ${ }^{137} \mathrm{Cs}$ - and ${ }^{22} \mathrm{Na}$-sources measured with the 1 t liquid argon detector, expressed as a function of the number of photoelectrons detected by 7 photomultipliers immersed in the liquid. The figure on the right shows the low energy distribution from the $511 \mathrm{keV}$ annihilation $\gamma$-rays.

ArDM equipped with 7 PMTs was filled for the first time in May 2009 with 2t of liquid argon (fiducial volume of $1 \mathrm{t}$ ) and kept full of liquid for more than one month without purification system. The slow component was measured to be constant during 20 days at $\tau_{2}=1.6 \pm 0.1 \mu \mathrm{s}$, in accord with the literature value [2]. Measurements of the light collection efficiency were performed with ${ }^{137} \mathrm{Cs}$ - and ${ }^{22} \mathrm{Na}$-sources. Figure 3 shows the energy distribution of the detected $\gamma$ 's, expressed as the number of photoelectrons (p.e.). The signal from the $662 \mathrm{keV} \gamma$ from Cs is clearly observed. For ${ }^{22} \mathrm{Na}$ measurements were performed by detecting the $511 \mathrm{keV}$ positron annihilation $\gamma$ 's in a $\mathrm{NaI}(\mathrm{Tl})$-detector, in coincidence with the $1.275 \mathrm{keV}$ transition, or by triggering on one of the 511 $\mathrm{keV} \gamma$ 's (see fig. 3). From these measurements one obtains about 0.5 p.e. / kev electron equivalent and therefore expects $\sim 1$ p.e./ kev with the completed detector (14 PMTs). Assuming a quenching factor of around $30 \%$ for nuclear recoils one then derives a light collection efficiency of $\sim 0.3$ p.e. / keV for WIMPs. This should be sufficient to detect $30 \mathrm{keV}$ nuclear recoils with good efficiency.

The charge readout will be also operational early in 2010. Following the extensive performance tests which can be most conveniently performed on the surface at CERN, we will then move the detector to an underground location, most likely the Canfranc Underground Laboratory.

\section{References}

[1] J. Angle et al., (XENON-10 collaboration), First results from the XENON10 Dark Matter Experiment at the Gran Sasso National Laboratory, Phys. Rev. Lett. 100 (2008) 021303; Z. Ahmed et al. (CDMS collaboration), Search for Weakly Interacting Massive Particles with the First Five-Tower Data from the Cryogenic Dark Matter Search at the Soudan Underground Laboratory, Phys. Rev. Lett. 102 (2009) 011301

[2] A. Hitachi et al., Effect of ionization density on the time dependence of luminescence from liquid argon and xenon, Phys. Rev. B 27 (1983) 5279.

[3] C. Amsler et al., Luminescence quenching of the triplet excimer state by air traces in gaseous argon, Journal of Instrumentation 3 (2008) P02001; V. Boccone et al., Development of wavelength shifter coated reflectors for the ArDM dark matter detector, Journal of Instrumentation 4 (2009) P06001. 